\title{
USO DE LOS RECURSOS DE LA BIODIVERSIDAD: ESTUDIO DE CASO DE LA OLEORRESINA DE COPAIBA (COPAIFERA SPP.) EN LA MEDICINA TRADICIONAL EN EL DEPARTAMENTO DEL META - COLOMBIA
}

\author{
USE OF BIODIVERSITY RESOURCES: CASE STUDY OF COPAIBA \\ OLEORRESINA (COPAIFERA SPP.) IN TRADITIONAL MEDICINE \\ IN THE META DEPARTMENT - COLOMBIA
}

\author{
${ }^{1}$ Luis Camilo Toro Chacon \\ ${ }^{2}$ Nicolás Rafael Francisco Robles Valderrama \\ ${ }^{3}$ Daniel Fernando Trigos Aguilera \\ ${ }^{4}$ Juan Manuel Trujillo González \\ ${ }_{5}^{5}$ Marco Aurelio Torres Mora \\ 1,2,3 Ingeniero Agroindustrial, Universidad de los Llanos, Instituto de Ciencias \\ Ambientales de la Orinoquia Colombiana ICAOC, Villavicencio - Colombia. \\ ${ }^{4}$ Ingeniero Agrónomo MSc. PhD. Universidad de los Llanos, Instituto de Ciencias \\ Ambientales de la Orinoquia Colombiana ICAOC, Grupo de Investigación \\ en Gestión Ambiental Sostenible - GIGAS. Villavicencio - Colombia. \\ ${ }^{5}$ Biólogo PhD, Universidad de los Llanos, Instituto de Ciencias Ambientales \\ de la Orinoquia Colombiana ICAOC, , Grupo de Investigación en \\ Gestión Ambiental Sostenible - GIGAS. Villavicencio - Colombia.

\footnotetext{
1 1luis.toro@unillanos.edu.co

2 nicolas.robles@unillanos.edu.co

${ }^{3}$ daniel.trigos@unillanos.edu.co

4 jtrujillo@unillanos.edu.co

${ }^{5}$ marcotorres@unillanos.edu.co
}

\section{RESUMEN}

Los recursos de la biodiversidad en los territorios son de vital importancia para el desarrollo socioeconómico y más aún cuando gran parte de su población depende de ellos, como es el caso de la Orinoquia Colombiana, región que cuenta con bosques compuestos por un género del árbol de Copaiba (Copaifera spp.) que origina un producto forestal no maderable, la oleorresina de copaiba o "aceite de palo". Este artículo aborda un comparativo entre el conocimiento tradicional y científico alrededor de las propiedades y usos dados a la oleorresina de Copaiba extraída del departamento del Meta, con base a una encuesta aplicada a 
comunidades rurales de los municipios de San Martín y Mapiripán, en el departamento del Meta - Colombia y publicaciones científicas actuales que abordan la temática específica. En el estudio se halló que gran parte de las propiedades atribuidas por la comunidad rural han sido investigadas con resultados que reafirman los usos tradicionales como medicamento en seres humanos, evidenciando también su utilización en animales. Se afirma entonces que la oleorresina de copaiba presenta principalmente propiedades benéficas para el sistema dérmico y gástrico. En su uso por parte de las comunidades rurales, especialmente población adulta, en un gran porcentaje (80\%), la ingiere en dosificaciones que van desde 3 a 20 gotas diarias, mientras que tan solo un $20 \%$ hace aplicación dérmica superficial. La variedad en el uso de la Oleorresina proveniente del género Copaiba y la necesidad de seguir profundizando en las propiedades medicinales que ofrece, pueden convertirse en una alternativa para su conservación y uso en áreas rurales donde se encuentra en el departamento del Meta.

Palabras clave: Medicinal, Orinoquia, Usos tradicionales, Aceite esencial, Aceite de palo.

\section{ABSTRACT}

The ecosystem services are of vital importance for the socioeconomic development of a region and even more when a large part of its population depends on them, as is the case of the region of the Colombian Orinoquia, and more specifically the department of Meta. The oleoresin of copaiba is a nontimber forest product indigenous to tropical zones found in the department of Meta, which has benefits for human health. The purpose of this article is to make Copaiba's oleoresin (Copaifera spp.) derived from supply and cultural services known as a socioeconomic alternative for the department of Meta through a comparison of its scientifically proven medicinal uses and those attributed by the rural community of the department. In the study it was found that a large part of the properties attributed by the rural community have already been studied with positive results, besides it was proved that it is not only used medicinally in humans but also in animals, with dosage ranging from 3 to 20 drops daily and dermal application. It is then stated that the oleoresin of copaiba has mainly beneficial properties for the dermal and gastric system, evidencing that a large percentage that consumes it is an adult population in dosages of 15 drops per day and superficial dermal applications.

Key words: Medicinal, Orinoquia, Traditional uses, Stick oil, Essential oil. 


\section{Resumen gráfico}
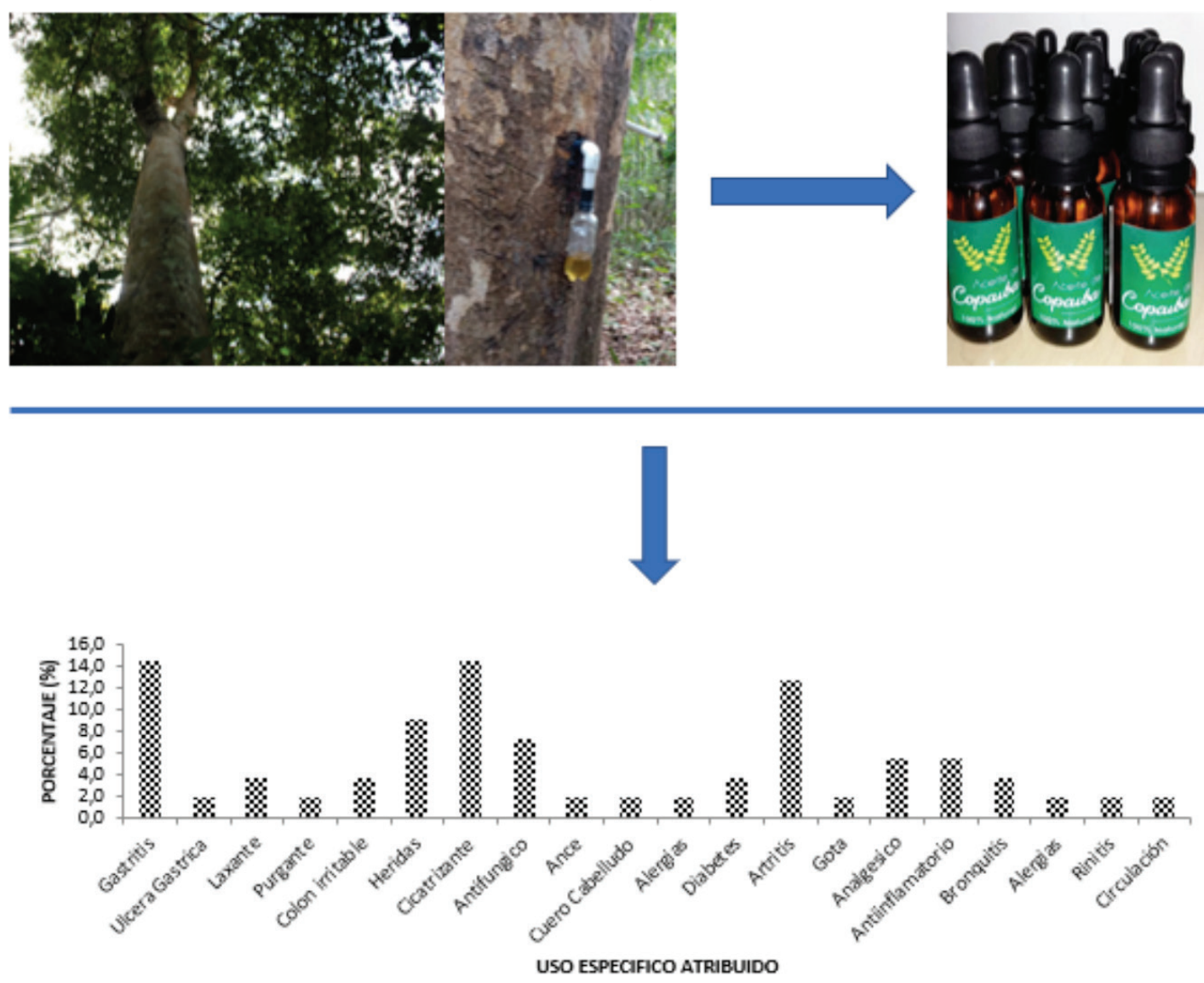

\section{INTRODUCCIÓN}

La biodiversidad es un importante productor de servicios ecosistémicos esenciales para el bienestar humano (Mori et al., 2017). Sin embargo, únicamente se han desarrollado estudios del $5 \%$ de ésta y y el mayor desafío ha sido saber cómo tener un acceso eficiente y eficaz, y valorizar la diversidad química natural (David et al., 2015). Un ejemplo de lo anterior es el género Copaifera spp., parte de la familia de las Fabaceae o leguminosas, donde los árboles, en su etapa adulta, son robustos con alturas que alcanzan de 25 a 40 metros y con diámetros hasta de 4 metros y distribuido en la región intertropical de América Latina y África Occidental (Junior y Pinto, 2002). García y Yamaguchi (2012), reportan que en el continente americano se distribuyen desde Argentina hasta México y que en Brasil se reporta el mayor número de especies con 20, de las cuales 17 son endémicas. En Colombia, el género se encuentra principalmente en la amazonia y parte de la Orinoquia, donde se han reportado tres especies Copaifera pubiflora (Benth) endémica del país y las especies Copaifera canime (Harms) y Copaifera officinalis (Jacq) (Bernal et al., 2011).

El principal producto de los árboles del género Copaifera spp. es la oleorresina o "aceite de palo", cuyas características son de un líquido viscoso extraído del tronco del árbol. Esta oleorresina es poco conocida comercialmente, sin embargo, en Brasil se han adelantado diversas investigaciones sobre su uso y aplicación (Garcia y Yamaguchi, 2012; Scur et al., 2016; Lucca et al., 2015). Para el caso específico de la Orinoquia Colombiana, las comunidades locales que 
cuentan con el recurso extraen la oleorresina de forma artesanal para comercializarla en la misma región con destino a diversos usos, principalmente enfocados en temas de salud humana. Con base en lo anterior, el propósito del presente estudio fue recopilar los usos tradicionales dados a la oleorresina de Copaifera spp por comunidades locales del departamento del Meta (Orinoquia colombiana) y compararlo con las investigaciones adelantadas a nivel mundial en esta temática, reportadas en la literatura científica.

\section{MATERIALES Y MÉTODOS}

La información primaria recolectada sobre el uso tradicional de la oleorresina de copaiba, se realizó mediante un instrumento de captura de información cualitativa tipo encuesta, que consistió en búsqueda sistemática de información en la que el investigador pregunta a los investigados sobre temas de interés (De Rada, 2012). Se consultaron temas sobre uso, dosis y asuntos generales de la oleorresina de copaiba a pobladores rurales del municipio de San Martín y municipios aledaños en el departamento del Meta que compran activamente el producto.

Para la recopilación de información secundaria se llevó a cabo una revisión literaria en la que se tomó como base de la información portales de búsqueda científica como Research Gate y Google Scholar, la búsqueda de los documentos consultados fue en los idiomas inglés, portugués y español. El principal criterio de inclusión de la búsqueda fue que las investigaciones se enfocaran en las especies Copaifera spp. y que trataran de la oleorresina; además los trabajos y artículos investigativos consultados fueron en $80 \%$ de los últimos 15 años. Como criterios de exclusión están aquellos trabajos que, aunque tratasen de Copaifera spp., no estuvieran orientados a la oleorresina y aquellas publicaciones que no contaran con fecha y autor.

\section{RESULTADOS Y DISCUSIÓN}

\section{Usos y aplicaciones tradicionales de oleo- rresina de Copaiba}

La identificación de los usos y aplicaciones de la oleorresina de Copaiba se llevó a cabo a través de una encuesta a compradores de este producto en mercado rural, en San Martín y municipios aledaños, en el departamento del Meta. En la figura 1, se presentan los resultados donde se evidencia que el $36,4 \%$ de la población encuestada lo usa como tratamiento dérmico, seguido de usos para tratamientos del sistema digestivo y articular con un $25,5 \%$ y $14,5 \%$ respectivamente.

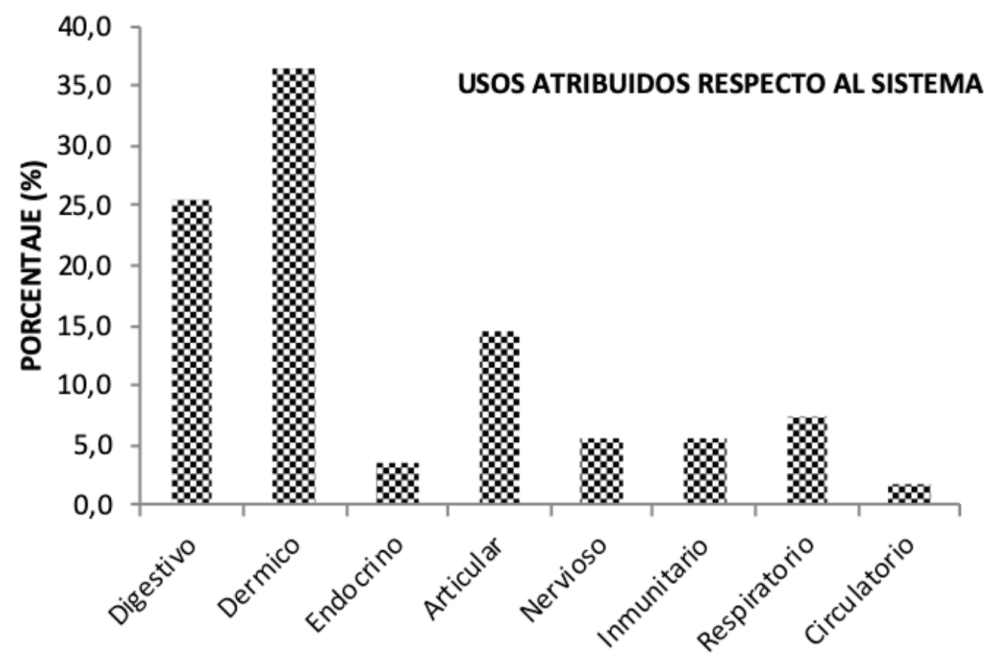

Figura 1. Usos medicinales atribuidos a la oleorresina de Copaiba por la comunidad rural del departamento del Meta. Fuente: Autoría propia. 
Los usos específicos más frecuentes dados por la comunidad rural a la oleorresina de copaiba (Figura 2.) es como tratamiento para la gastritis
$(15 \%)$, cicatrices $(15 \%)$, artritis $(13 \%)$, heridas (9\%) y antifúngico (7\%).

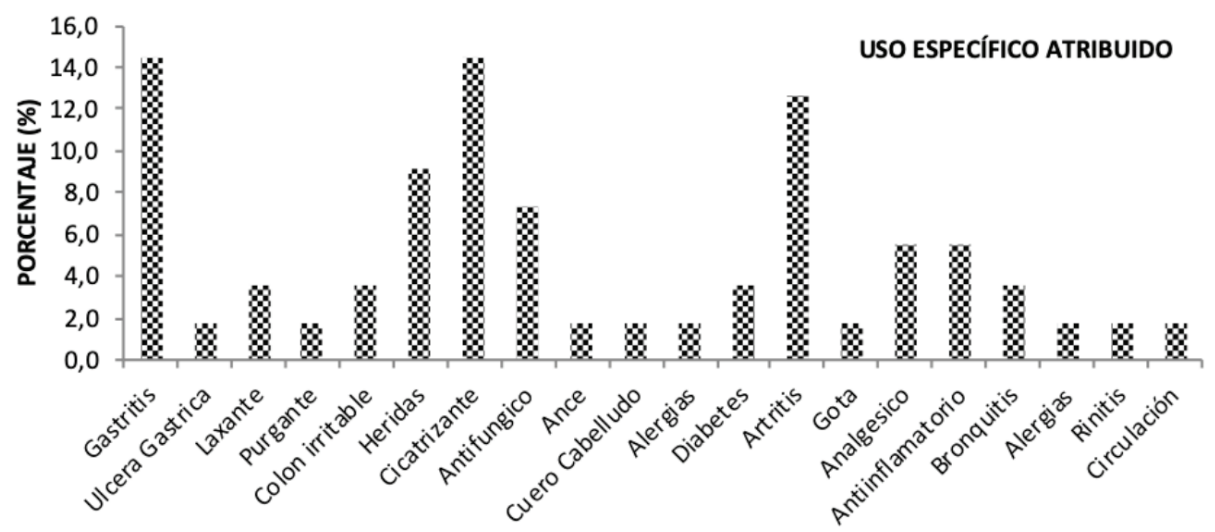

Figura 2. Usos medicinales específicos atribuidos por la comunidad rural del departamento del Meta a la oleorresina de copaiba.

Fuente de: Autoría propia.

Con respecto al modo de uso, el $80 \%$ de los encuestados coincidieron que la mejor forma de aplicación de este producto es por vía oral, de los cuales el $30 \%$ ingiere 15 gotas/día, y el $25 \%$ ingiere 3 gotas/día; esto coincide con los usos medicinales atribuidos puesto que el consumo de 15 gotas/día, según palabras de los encuestados, se hace con el fin de obtener tratamientos en el sistema digestivo específicos como gastritis, laxante y colon irritable. El $20 \%$ restante de la población encuestada hace uso dérmico de la oleorresina de copaiba para tratamientos específicos como cicatrizante, para heridas y antifúngico.
Finalmente, en cuanto al tipo de población que hace uso del producto, el 66,7\% del total de los encuestados expresó que son los adultos los principales consumidores, sin embargo, un $14,8 \%$ manifiesta que en su hogar hacen uso de la oleorresina personas de todas las edades, y un 3,7\% manifestó que son los menores quienes la consumen. Adicionalmente, se identificó que los compradores de la oleorresina también la utilizan para tratar a sus animales, ya que el $14,8 \%$ de los encuestados así lo manifestaron (Figura 3).

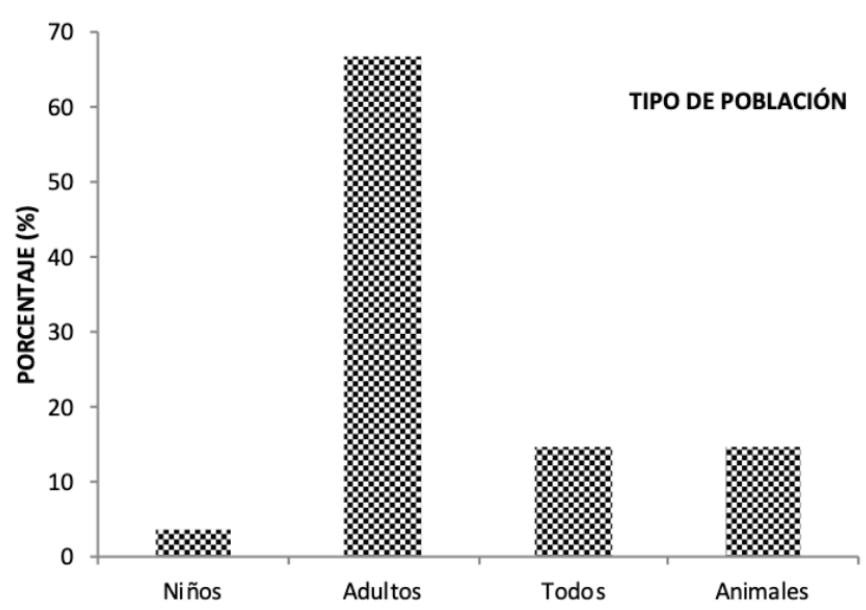

Figura 3. Tipo de población que consume la oleorresina de copaiba. 
Análisis comparativo usos tradicionales vs. reportes en literatura científica

En la búsqueda de información secundaria y en concordancia con los criterios de inclusión y exclusión se encontraron 42 estudios, de los cuales se excluyeron 9, y los 33 restantes fueron artículos científicos y tesis de grado. Para la información primaria se encuestó a 22 personas habitantes del área rural de los municipios Mapiripán y San Martín. De manera general se encontró que las comunidades locales utilizan la oleorresina de Copaiba para tratar 20 afectaciones de la salud, tales como enfermedades asociadas al sistema dérmico en un $36,4 \%$, Digestivo 25,5 \%, articular 12,7 \%, respiratorio $7,3 \%$ entre otros, las dosis para estos tratamientos varían entre 3 y 20 gotas al día y el $66,7 \%$ de los consumidores son adultos. Por su parte, Arroyo-Acevedo et al. (2011) y Arroyo et al. (2009), mencionan que el estudio del aceite de copaiba para el beneficio humano directo e indirecto ha tenido diferentes enfoques investigativos principalmente en Brasil, donde se ha reportado su uso tradicional desde el siglo XVI por los habitantes nativos del norte y noreste del país, como antiinflamatorio, antitumoral, antiséptico urinario, contra enfermedades de la piel, úlceras y cicatrizante de heridas.

Las comunidades rurales mencionan que el $25,5 \%$ de los usos esta orientados a tratar enfermedades relacionadas con el sistema digestivo tales como: gastritis, ulcera gástrica, colon irritable y con uso laxante y purgante. Por su parte, en la literatura se reportan estudios también relacionados con el sistema digestivo como tratamiento para la gastritis, la ulcera gástrica y el cáncer gástrico; uno de los estudios para tratar la ulcera gástrica fue llevado a cabo con la oleorresina de la especie C. langsdorffi administrado por vía oral con diferentes concentraciones en ratas con lesiones gástricas, presentando resultados positivos en todas las pruebas, esto destaca el potencial gastroprotector de la oleorresina (Paiva et al., 1998); otro estudio enfocado para tratamiento de gastritis fue llevado a cabo con la oleorresina de la especie $C$. officinalis también en ratas, y presentó una eficacia de $100 \%$ como citoprotector, superando al omeprazol (97.8\%), verificado mediante cortes histológicos, mostrando resultados efectivos nuevamente como agente gastroprotector (Arroyo et al., 2009). Un estudio para el tratamiento del cáncer gástrico fue llevado a cabo con ácido Kaurenoico obtenido a partir de la oleorresina de copaiba y mostró que induce daño en el ADN y aumenta la frecuencia de micronúcleos de forma dependiente de la dosis en las células del cáncer gástrico, con una genotoxicidad significativa observada por encima de la concentración de $5 \mu \mathrm{g} / \mathrm{ml}$ y además, este compuesto disminuye el ciclo celular y la apoptosis en células del cáncer gástrico (Dos Santo Cardoso et al., 2017).

Entre los usos medicinales se encuentran varios enfocados al sistema dérmico, representando estos un total del $36,4 \%$ de usos mencionados por las comunidades locales, donde los asocian al tratamiento para heridas, cicatrizante, antifúngico, acné, cuero cabelludo y alergias. Por su parte, en la literatura se reportan estudios de usos medicinales similares a los reportados por las comunidades locales, tales como: tratamiento de la leishmaniasis, heridas cutáneas, cicatrización y antifúngico (Santos et al., 2008; Kvist et al., 2006; Santos et al., 2011; Santos et al., 2012); mediante estos estudios, aunque no se ha identificado claramente el mecanismo de acción de la oleorresina sobre el parásito leishmania, es una opción viable y rápida de tratamiento, segura y más económica que los mecanismos tradicionalmente utilizados (Santos et al. 2011). Otro estudio llevado a cabo con la oleorresina de la especie $C$. Langsdorffii en heridas cutáneas inducidas a ratas, indican el efecto beneficioso 
en el tratamiento y cicatrización de las heridas, lo que justifica su uso tradicional para tal fin (Paiva et al., 2002); Otro estudio llevado a cabo con la oleorresina de copaiba en ratas se enfoca a evaluar la capacidad de cicatrización en heridas alveolares que tiene esta oleorresina concluyendo que la administración sistémica de la oleorresina de copaiba conduce a una mejor curación ósea alveolar, sin embargo, la aplicación de la oleorresina sobre el tejido conectivo debe considerarse cuidadosamente, con respecto a la cicatrización de toda la herida (Dias-da-Silva, et al., 2013); Un último estudio que demuestra la influencia de la oleorresina en los tratamiento a afectaciones del sistema dérmico se llevó a cabo con la oleorresina de la especie C. Langsdorffii contra diferentes hongos que afectan los tejidos queratinizados tanto en humanos como en animales, demostrando mediante los resultados un daño físico y alteraciones morfológicas tales como compresión e hifas agrupadas en la estructura de los hongos expuestos a la acción de la oleorresina. Esto confirma los beneficios de la aplicación de oleorresina extraída de copaiba en el tratamiento de la dermatofitosis, tanto en humanos como en animales (ZimmermamFranco et al., 2013). Entre los usos medicinales tradicionales enfocados al sistema dérmico que le da la comunidad rural a la oleorresina pero que no han sido estudiados científicamente, se tienen el tratamiento para el acné, alergias cutáneas y cuero cabelludo.

Entre los usos medicinales se encuentra uno enfocado al sistema endocrino, más específicamente al tratamiento contra la diabetes, representando este un total del $3,6 \%$ de las respuestas de los usos tradicionales que le da la comunidad rural a la oleorresina. De otro lado, en la literatura se encuentra un estudio relacionado indirectamente con el tratamiento para la diabetes, la investigación se enfoca en la mejor capacidad de curación de heridas que tiene la oleorresina de copaiba versus una pomada usada para estas lesiones, por lo cual lo sugiere como una buena opción para su uso en pacientes diabéticos (Amorim et al., 2017).

Otro de los principales usos medicinales que ha tenido el aceite de copaiba a nivel tradicional en el sistema inmunitario y articular es para la artritis inflamatoria e inflamaciones en el cuerpo humano en general, ya que el $16,4 \%$ de las personas encuestadas así lo confirman. La población rural no se ha equivocado al hacer esta apreciación y atribuirle estos usos a la oleorresina, ya que en un experimento realizado en el 2016, a 3 grupos de ratas se les suministraron 3 tratamientos: indometacina, cloruro de sodio y aceite de copaiba, para luego inducirles un edema por medio de un agente inflamatorio (carragenina) y comparar el efecto de cada tratamiento, resultando la oleorreisna de copaiba con un efecto muy similar al de la indometacina al momento de evitar la formación de edemas (Canchuja et al., 2016). También se confirma el comportamiento antiinflamatorio del aceite de copaiba al momento de tratar en un experimento el edema en patas de ratas con oleorresina extraída de la especie Copaifera multijuga Hayne, obteniendo como resultado que el aceite presenta una actividad antiinflamatoria a través de la inhibición de las vías histaminérgicas y serotoninérgicas (De Matos Gomes et al., 2010). Específicamente, otro estudio llevado a cabo con oleorresina de la especie $C$. reticulata a ratas con artritis inducida por adyuvante reveló que la oleorresina de copaiba presentó acciones sistémicas antiinflamatorias y antioxidantes en ratas artríticas, sin embargo, estos efectos beneficiosos fueron contrarrestados por modificaciones perjudiciales en el metabolismo hepático y la morfología de las ratas de control sanas (Castro Ghizoni et al., 2017). 
Además de las propiedades benéficas de la oleorresina de copaiba al sistema inmunitario y articular nombradas anteriormente, un $1,8 \%$ de la población rural encuestada afirma utilizar la oleorresina como tratamiento para la enfermedad de la gota, hecho que aún no se ha corroborado científicamente.

Otra de las propiedades medicinales nombradas por la comunidad rural en la encuesta aplicada fue su efecto analgésico, ya sea como antinociceptivo o ansiolítico, con un 5,5\% de la población encuestada que lo confirma. Resulta de gran relevancia en la actualidad encontrar compuestos analgésicos naturales en reemplazo de los analgésicos sintéticos que pueden llegar a producir adicción y problemas estomacales, e incluso en reemplazo de analgésicos narcóticos usados en casos especiales., motivo que ha llevado a investigaciones en el aceite de la especie $C$. dukei Dwyer para uso analgésico en ratas a diferentes concentraciones, presentando resultados positivos de inhibición del proceso de retorcimiento, lo que indica que la oleorresina tiene actividades tópicas analgésicas (Carvalho et al., 2005). Se han llevado a cabo de igual forma dos estudios con la oleorresina de copaiba para evaluar el efecto antinociceptivo que esta posee obteniendo resultados positivos, el primero adelantado con C. Multijuga demostró un efecto antinociceptivo probablemente mediado por receptores opioides ya que la posterior administración de naloxona inhibió tal efecto (De Matos Gomes et al. 2010), el otro estudio llevado a cabo mediante una comparación de las oleorresinas de Copaifera multijuga Hayne y Copaifera reticulata Ducke, demostraron en primer lugar que los aceites de Copaiba no desarrollan efectos tóxicos en el organismo e indicaron también que demuestran un efecto antinociceptivo periférico y central, el cual puede ser útil en el tratamiento de trastornos algésicos (Gomes et al. 2007). Otro análisis de la oleorresina de copaiba proveniente de la Copaifera reticulata Ducke con la finalidad de comprobar el efecto ansiolítico que esta podía llegar a presentar aplicada experimentalmente a ratas, produjo un efecto ansiolítico positivo sin afectar adversamente los niveles generales de actividad (Curio et al. 2009).

De los usos medicinales atribuidos por la comunidad rural, un $7,3 \%$ de la población encuestada le atribuye a la oleorresina de copaiba propiedades benéficas para el sistema respiratorio como tratamiento para la gripa, alergias respiratorias y la rinitis, propiedades que no han sido corroboradas científicamente, pero que podrían asociarse a los efectos antimicrobianos confirmados por investigaciones del potencial antimicrobiano de la oleorresina proveniente de géneros de copaifera spp. y de la $C$. langsdorffi, la cual esterilizada en autoclave puede considerarse como una fuente de agente potencial contra la infección o para la conservación de alimentos, ya que inhibe el crecimiento de bacilos gram positivos como la Listeria monocytogenes (Pieri, 2010).

Para el caso de la isquemia, detención o disminución en la circulación sanguínea a través de las arterias de una determinada zona del cuerpo (Ramos-Gallardo y Miranda, 2014), el 1,8\% de la población rural encuestada dice usar la oleorresina de copaiba como tratamiento para esta afectación del sistema circulatorio, propiedad que ha sido estudiada científicamente también. a partir de $C$. langsdorffi, cuya oleorresina fue evaluada en su efecto para el daño intestinal asociado con isquemia/reperfusión a dos grupos de ratas albinas a las que se les aplicó 200 y 400 mg/kg respectivamente, y cuyos resultados se expresan con atenuaciones significativas en los aumentos asociados con las actividades de mieloperoxidasa, malondialdehído y catalasa, al igual que en el nivel de 
nitrito, indicando que la oleorresina de esa especie en particular tiene una acción protectora contra el daño del tejido intestinal inducido por la isquemia/reperfusión (Paiva et al., 2004).

Además de los usos medicinales, el 1,8\% de la población rural encuestada afirma que la oleorresina de copaiba tiene propiedades insecticidas. Un estudio con el objetivo de reemplazar los insecticidas $100 \%$ sintéticos con mezclas de insecticidas botánicos y sintéticos, encontró que la toxicidad de la oleorresina aumentó sustancialmente al adicionarle insecticidas sintéticos, especialmente con mezclas más bajas con beta-cipermetrina, metomilo y triazofos (Almeida et al., 2017), lo que corrobora el uso como insecticida dado por parte de la población rural a la oleorresina de copaiba.

A nivel mundial son claros los esfuerzos investigativos que se han hecho para contrarrestar los efectivos negativos y mortales del cáncer, donde la oleorresina de copaiba ha sido estudiada como alternativa natural para el tratamiento contra este letal mal; es necesario aclarar que en la encuesta desarrollada la población rural no nombro la propiedad antitumoral. En pruebas hechas a diferentes tipos de cáncer con la oleorresina se han comprobado resultados positivos y uno negativo; en cuanto a los primeros resultados, se demostró que la oleorresina de $C$. multijuga y sus fracciones tienen actividad antitumoral en la línea celular de melanoma en modelos in vivo e in vitro, reduciendo el crecimiento del tumor en un $58 \%$ y el peso del tumor en un $76 \%$ (Lima et al. 2003). En otro de los resultados positivos con aceite de $C$. multijuga se encontró que el tumor ascítico de Ehrlich (EAT) redujo su volumen, demostrando así su efecto neoplásico después de su administración oral (de Matos Gomes et al. 2008). En cuanto al resultado negativo como agente antitumoral, se suministró a ratas con carcinoma Walker 256 oleorresina de $C$. officinalis, observándose un potencial inhibitorio negativo del $70 \%$ y estímulo al crecimiento del tumor (Brito et al. 2010).

Otros estudios adelantados en los últimos años tienen que ver con investigaciones acerca de la capacidad mutagénica de la oleorresina de diferentes especies de Copaibas. De manera específica para la especie C. dukei Dwyer utilizando tres diferentes concentraciones y tres diferentes intervalos de tiempo en ratas, presentó resultados negativos, debido a que no se produjeron efectos mutagénicos sobre las células de la médula ósea ni en los reticulocitos periféricos evaluados por aberraciones cromosómicas (Maistro et al., 2005). Otro estudio de evaluación del potencial mutagénico con la especie $C$. langsdorffii Desfon en ratas con diferentes concentraciones de oleorresina y diferentes tiempos de administración oral, obtuvo en cambio resultados positivos en cuanto a la potencialidad mutagénica, ya que, se observó un aumento significativo de frecuencia de micronúcleos en eritrocitos policromáticos (EPCMN) con frecuencia máxima a las 24 horas (Chen-Chen y Sena, 2002).

La oleorresina de copaiba a pesar de ser conocida por su amplia variedad de propiedades benéficas para el ser humano también presenta algunos efectos negativos que han sido evaluados con el fin de no hacer un uso indiscriminado del producto. Estudios acerca del efecto antiproliferativo que tiene la oleorresina extraída de la especie C. duckei, tratando un grupo de ratas con el producto antes de someterlas a una cirugía en el hígado, se encontró un aumento en el consumo de oxígeno mitocondrial acoplado a la síntesis de ATP, dando lugar a una disminución significativa en la relación del control respiratorio, lo que se traduce en un desacoplamiento de la fosforilación oxidativa en la mitocondria y por ende una disminución en la proliferación hepatocelular, demostrando el efecto tóxico que tiene 
este producto (Casto-e-Silva et al., 2004). Adicionalmente se conoce el efecto embriotóxico de la oleorresina de $C$. reticulata, donde a través de una investigación realizada en ratas Wistar embarazadas, a las que se les suministró en diferentes concentraciones, se determinó que fue tóxica para la madre reduciendo el consumo de alimentos y aumentando el peso, al igual que provocó menor peso corporal y mayor incidencia de variaciones del esqueleto fetal, ambos resultados a partir de concentraciones de $500 \mathrm{mg} / \mathrm{kg} /$ día (Sachetti et al., 2011).

\section{CONCLUSIONES}

A partir de la revisión literaria se pudo corroborar que el conocimiento tradicional de las comunidades rurales de San Martín y Mapiripá, Meta, Colombia, respecto a las propiedades de la oleorresina de copaiba, es acertado. Al consultar las diferentes fuentes científicas, se encontró que, para la mayoría de los usos atribuidos por las comunidades, existe al menos una investigación que lo respalda.

Por medio de la comparación que se desarrolló en el presente trabajo entre el conocimiento tradicional y el científico, se presenta la necesidad de seguir profundizando en nuevas investigaciones acerca del potencial medicinal de estas sustancias naturales que provienen de la diversidad de especies de este género, lo que puede significar que haya variaciones en la composición de la oleorresina y a su vez en las propiedades de la misma. A raíz de lo anterior, se sugiere que los futuros estudios estén orientados de forma progresiva desde la botánica hasta la gestión ambiental del recurso natural, como estrategia para lograr un aprovechamiento sostenible por parte de las comunidades rurales poseedoras de áreas donde está establecido el género Copaifera spp, especialmente en la Orinoquia colombiana.

\section{LITERATURA CITADA}

Alcaldía de Mapiripán. (2016) Plan de desarrollo "La Fuerza del Pueblo 2016 - 2019". Recuperado de: [https://ceo.uniandes.edu.co/images/Documentos/Plan_de_Desarrollo-_Mapirip\%C3\%A1n.pdf]

Almeida, W. A. D., Silva, I. H. I. D., Santos, A. C. V. D., Barros Junior, A. P., \& Sousa, A. H. D. (2017). Potentiation of Copaíba Oil-resin with Synthetic Insecticides to Control of Fall Armyworm. Revista caatinga, 30(4), 1059-1066.

Amorim, J. L., de Barros Figueiredo, J., Amaral, A. C. F., de Oliveira Barros, E. G., Palmero, C., MPalantinos, M. A., ... \& Basso, S. L. (2017). Wound healing properties of Copaifera paupera in diabetic mice. PloS one, 12(10), e0187380.

Arroyo, J., Almora, Y., Quino, M., Martínez, J., Condorhuamán, M., Flores, M., \& Bonilla, P. (2009). Copaifera officinalis oil cytoprotector and antisecretory effects in induced gastric lesions in rats. Anales de la Facultad de Medecina, 70(2), 89-96.

Arroyo-Acevedo, J., Quino-Florentini, M., Martínez-Heredia, J., Almora-Pinedo, Y., Alba-González, A., \& Condorhuamán-Figueroa, M. (2011, April). Efecto cicatrizante del aceite de Copaifera officinalis (copaiba), en pacientes con úlcera péptica. En Anales de la Facultad de Medicina (Vol. 72, No. 2, pp. 113-117). UNMSM. Facultad de Medicina.

Bernal, H. Y., Martínez, G., Sánchez, Q., \& FelipeEd, G. (2011). Pautas para el conocimiento, conservación y uso sostenible de las plantas medicinales nativas en Colombia. Estrategia nacional para la conservación de plantas.

Brito, N. M. B., Brito, M. V. H., Carvalho, R. D. K. V., Matos, L. T. D. M. B., Lobato, R. C., Correa, S. C., \& Brito, R. B. (2010). The effect of copaiba balsam on Walker 256 carcinoma inoculated into the vagina and uterine cervix of female rats. Acta cirurgica brasileira, 25(2), 176-180.

Canchuja, F. E. A., Lazo, K. A., \& Contreras, C. (2016). Estudio experimental comparativo del efecto analgésico y antiinflamatorio de Copaiba frente a morfina e indometacina. Revista de la facultad de medicina humana, 14(1).

Carvalho, J. C. T., Cascon, V., Possebon, L. S., Morimoto, M. S. S., Cardoso, L. G. V., Kaplan, M. A. C., \& Gilbert, B. (2005). Topical antiinflammatory and analgesic activities of Copaifera duckei Dwyer. Phytotherapy Research, 19(11), 946-950. 
Castro Ghizoni, C. V., Arssufi Ames, A. P., Lameira, O. A., Bersani Amado, C. A., Sá Nakanishi, A. B., Bracht, L., ... \& Comar, J. F. (2017). Anti-Inflammatory and Antioxidant Actions of Copaiba Oil Are Related to Liver Cell Modifications in Arthritic Rats. Journal of cellular biochemistry, 118(10), 3409-3423.

Castro-e-Silva, O., Zucoloto, S., Ramalho, F. S., Ramalho, L. N., Reis, J., Bastos, Á. A., \& Brito, M. V. (2004). Antiproliferative activity of Copaifera duckei oleoresin on liver regeneration in rats. Phytotherapy Research, 18(1), 92-94.

Chen-Chen, L., \& Sena, M. A. (2002). Atividade tóxica e mutagênica do óleo de copaíba (Copaifera langsdorfii Desfon) em camundongos. Rev. Bras. Plant Med, 5, 37-40.

Curio, M., Jacone, H., Perrut, J., Pinto, Â. C., Valdir Filho, F. V., \& Silva, R. C. (2009). Acute effect of Copaifera reticulata Ducke copaiba oil in rats tested in the elevated plus-maze: an ethological analysis. Journal of Pharmacy and Pharmacology, 61(8), 1105-1110.

De Matos Gomes, N., de Moraes Rezende, C., Fontes, S. P., Hovell, A. M. C., Landgraf, R. G., Matheus, M. E., ... \& Fernandes, P. D. (2008). Antineoplasic activity of Copaifera multijuga oil and fractions against ascitic and solid Ehrlich tumor. Journal of ethnopharmacology, 119(1), 179-184.

De Matos Gomes, N., de Rezende, C. M., Fontes, S. P., Matheus, M. E., da Cunha Pinto, A., \& Fernandes, P. D. (2010). Characterization of the antinociceptive and anti-inflammatory activities of fractions obtained from Copaifera multijuga Hayne. Journal of ethnopharmacology, 128(1), 177-183.

David, B., Wolfender, J. L., \& Dias, D. A. (2015). The pharmaceutical industry and natural products: historical status and new trends. Phytochemistry Reviews, 14(2), 299-315.

De Rada, V. D. (2012). Ventajas e inconvenientes de la encuesta por Internet. Papers: revista de sociología, 97(1), 193-223.

Dias-da-Silva, M. A., Pereira, A. C., Marin, M. C., \& Salgado, M. A. (2013). The influence of topic and systemic administration of copaiba oil on the alveolar wound healing after tooth extraction in rats. Journal of clinical and experimental dentistry, 5(4), e169.

Dos Santos Cardoso, P. C., da Rocha, C. A. M., Leal, M. F., de Oliveira Bahia, M., Alcântara, D. D. F. Á., dos Santos, R. A., ... \& do Ó Pessoa, C. (2017). Effect of diterpenoid kaurenoic acid on genotoxicity and cell cycle progression in gastric cancer cell lines. Biomedicine \& Pharmacotherapy, 89, 772-780.

Garcia, R. F., \& Yamaguchi, M. H. (2012). Óleo de copaíba e suas propriedades medicinais: revisão bibliográfica. Saúde e Pesquisa, 5(1).

Gomes, N. M., Rezende, C. M., Fontes, S. P., Matheus, M. E., \& Fernandes, P. D. (2007). Antinociceptive activity of Amazonian Copaiba oils. Journal of Ethnopharmacology, 109(3), 486-492.

Junior, V. V., \& Pinto, A. C. (2002). O Gênero Copaifera L. Quim. Nova, 25(2), 273-286. Kvist, L. P., Christensen, S. B., Rasmussen, H. B., Mejia, K., \& Gonzalez, A. (2006). Identification and evaluation of Peruvian plants used to treat malaria and leishmaniasis. Journal of ethnopharmacology, 106(3), 390-402.

Kvist, L. P., Christensen, S. B., Rasmussen, H. B., Mejia, K., \& Gonzalez, A. (2006). Identification and evaluation of Peruvian plants used to treat malaria and leishmaniasis. Journal of ethnopharmacology, 106(3), 390-402.

Lima, S. R., Junior, V. F. V., Christo, H. B., Pinto, A. C., \& Fernandes, P. D. (2003). In vivo and in vitro studies on the anticancer activity of Copaifera multijuga Hayne and its fractions. Phytotherapy Research, 17(9), 1048-1053.

Lucca, L. G., de Matos, S. P., Borille, B. T., Dias, D. D. O., Teixeira, H. F., Veiga Jr, V. F., ... \& Koester, L. S. (2015). Determination of $\beta$-caryophyllene skin permeation/retention from crude copaiba oil (Copaifera multijuga Hayne) and respective oil-based nanoemulsion using a novel HS-GC/MS method. Journal of pharmaceutical and biomedical analysis, 104, 144-148.

Maistro, E. L., Carvalho, J. C. T., Cascon, V., \& Kaplan, M. A. C. (2005). In vivo evaluation of the mutagenic potential and phytochemical characterization of oleoresin from Copaifera duckei Dwyer. Genetics and Molecular Biology, 28(4), 833-838.

Mori, A. S., Lertzman, K. P., \& Gustafsson, L. (2017). Biodiversity and ecosystem services in forest ecosystems: a research agenda for applied forest ecology. Journal of Applied Ecology, 54(1), 12-27

Organización de las Naciones Unidas para la Educación, la Ciencia y la Cultura (UNESCO). (2017). 
Sistemas de Conocimientos Locales e Indígenas. Recuperado de: http://www.unesco.org/new/ es/natural-sciences/priority-areas/links/related-information/what-is-local-and-indigenousknowledge/

Paiva, L. A. F., de Alencar Cunha, K. M., Santos, F. A., Gramosa, N. V., Silveira, E. R., \& Rao, V. S. N. (2002). Investigation on the wound healing activity of oleoresin from Copaifera langsdorffi in rats. Phytotherapy Research: An International Journal Devoted to Pharmacological and Toxicological Evaluation of Natural Product Derivatives, 16(8), 737-739.

Paiva, L. A. F., Gurgel, L. A., Campos, A. R., Silveira, E. R., \& Rao, V. S. N. (2004). Attenuation of ischemia/reperfusion-induced intestinal injury by oleoresin from Copaifera langsdorffii in rats. Life Sciences, 75(16), 1979-1987.

Paiva, L. A. F., Rao, V. S. N., Gramosa, N. V., \& Silveira, E. R. (1998). Gastroprotective effect of Copaifera langsdorffii oleoresin on experimental gastric ulcer models in rats. Journal of ethnopharmacology, 62(1), 73-78.

Pieri, F. A., José, R. M., Galvão, N. N., Nero, L. A., \& Moreira, M. A. S. (2010). Antimicrobial activity of autoclaved and non autoclaved copaiba oil on Listeria monocytogenes. Ciência Rural, 40(8), 1797-1801.

Ramos-Gallardo, G., \& Altamirano, A. M. (2014). Importancia del daño isquemia-reperfusión en cirugía plástica. Cirugía Plástica, 24(1), 57-60.

Sachetti, C. G., de Carvalho, R. R., Paumgartten, F. J., Lameira, O. A., \& Caldas, E. D. (2011). Developmental toxicity of copaiba tree (Copaifera reticulata Ducke, Fabaceae) oleoresin in rat. Food and Chemical Toxicology, 49(5), 1080-1085.
Santos, A. O., Costa, M. A., Ueda-Nakamura, T., Dias-Filho, B. P., da Veiga-Júnior, V. F., de Souza Lima, M. M., \& Nakamura, C. V. (2011). Leishmania amazonensis: effects of oral treatment with copaiba oil in mice. Experimental parasitology, $129(2), 145-151$.

Santos, A. O., Ueda-Nakamura, T., Dias Filho, B. P., da Veiga Junior, V. F., \& Nakamura, C. V. (2012). Copaiba oil: an alternative to development of new drugs against leishmaniasis. Evidence-Based Complementary and Alternative Medicine, 2012.

Santos, A. O., Ueda-Nakamura, T., Dias Filho, B. P., Junior, V. F. V., Pinto, A. C., \& Nakamura, C. V. (2008). Effect of Brazilian copaiba oils on Leishmania amazonensis. Journal of ethnopharmacology, 120(2), 204-208.

Scur, M. C., Pinto, F. G. S., Pandini, J. A., Costa, W. F., Leite, C. W., \& Temponi, L. G. (2016). Antimicrobial and antioxidant activity of essential oil and different plant extracts of Psidium cattleianum Sabine. Brazilian Journal of Biology, 76(1), 101-108.

Zimmermam-Franco, D. C., Bolutari, E. B., Polonini, H. C., do Carmo, A. M. R., das Graças AM Chaves, M., \& Raposo, N. R. (2013). Antifungal activity of Copaifera langsdorffii Desf oleoresin against dermatophytes. Molecules, 18(10), 12561-12570.

\section{AGRADECIMIENTOS}

Al apoyo financiero del Instituto de Ciencias Ambientales de la Orinoquia Colombiana - ICAOC de la Universidad de los Llanos y al productor de la oleorresina de copaiba Wilson Gutiérrez del municipio de Mapiripán - Meta por toda la información suministrada.
Conflicto de Intereses Los autores declaran no tener ningún conflicto de intereses 